\title{
Two-dimensional solar spectropolarimetry with the KIS/IAA Visible Imaging Polarimeter
}

\author{
C. Beck ${ }^{1,2}$, L. R. Bellot Rubio ${ }^{3}$, T. J. Kentischer ${ }^{4}$, A. Tritschler ${ }^{5}$, and J. C. del Toro Iniesta ${ }^{3}$ \\ 1 Instituto de Astrofísica de Canarias (IAC), C/Vía Láctea S/N, 38205 La Laguna, Tenerife, Spain \\ e-mail: cbeck@iac.es \\ 2 Departamento de Astrofísica, Universidad de La Laguna, 38205 La Laguna, Tenerife, Spain \\ 3 Instituto de Astrofísica de Andalucía (CSIC), Apdo. de Correos 3004, 18080 Granada, Spain \\ e-mail: [lbellot;jti]@iaa.es \\ 4 Kiepenheuer-Institut für Sonnenphysik, Schöneckstr. 6, 79104 Freiburg, Germany \\ e-mail: tk@kis.uni-freiburg.de \\ 5 National Solar Observatory/Sacramento Peak ${ }^{\star}$, PO Box 62, Sunspot, NM 88349, USA \\ e-mail: atritschler@nso.edu
}

Received 9 October 2009 / Accepted 28 June 2010

\section{ABSTRACT}

\begin{abstract}
Context. Spectropolarimetry at high spatial and spectral resolution is a basic tool to characterize the magnetic properties of the solar atmosphere.

Aims. We introduce the KIS/IAA Visible Imaging Polarimeter (VIP), a new post-focus instrument that upgrades the TESOS spectrometer at the German Vacuum Tower Telescope (VTT) into a full vector polarimeter. VIP is a collaboration between the Kiepenheuer Institut für Sonnenphysik (KIS) and the Instituto de Astrofísica de Andalucía (IAA-CSIC).

Methods. We describe the optical setup of VIP, the data acquisition procedure, and the calibration of the spectropolarimetric measurements. We show examples of data taken between 2005 and 2008 to illustrate the potential of the instrument.

Results. VIP is capable of measuring the four Stokes profiles of spectral lines in the range from 420 to $700 \mathrm{~nm}$ with a spatial resolution better than 0.'5. Lines can be sampled at 40 wavelength positions in $60 \mathrm{~s}$, achieving a noise level of about $2 \times 10^{-3}$ with exposure times of $300 \mathrm{~ms}$ and pixel sizes of 0 ' $^{\prime} 17 \times 0{ }^{\prime} \cdot 17(2 \times 2$ binning). The polarization modulation is stable over periods of a few days, ensuring high polarimetric accuracy. The excellent spectral resolution of TESOS allows the use of sophisticated data analysis techniques such as Stokes inversions. One of the first scientific results of VIP presented here is that the ribbon-like magnetic structures of the network are associated with a distinct pattern of net circular polarization away from disk center.

Conclusions. VIP performs spectropolarimetric measurements of solar magnetic fields at a spatial resolution that is only slightly worse than that of the Hinode spectropolarimeter, while providing a 2D field field of view and the possibility to observe up to four spectral regions sequentially with high cadence. VIP can be used as a stand-alone instrument or in combination with other spectropolarimeters and imaging systems of the VTT for extended wavelength coverage.
\end{abstract}

Key words. instrumentation: polarimeters - Sun: photosphere - magnetic fields

\section{Introduction}

Measurements of the solar magnetic field and the determination of the thermal and kinematic properties of the magnetized atmosphere is a persistently challenging goal. The combination of high spectral and angular resolution is of vital importance for an accurate characterization of the atmospheric parameters in different structures, from the tiny magnetic elements of the quiet Sun to the largest sunspots. However, only a few ground-based instruments are capable of high-precision polarimetric observations with an angular resolution of $0 . ' 5$ or better.

Two different concepts have been followed to obtain measurements of the full Stokes vector at high spectral resolution: imaging and grating (slit-based) spectropolarimeters. Modern imaging spectropolarimeters employ single (see, e.g., Mickey et al. 1996) or multiple Fabry-Pérot etalons (FPIs) for fast tuning and high transmission. Several FPI spectropolarimeters are operated at present: the Göttingen spectropolarimeter (GFPI, Bello González \& Kneer 2008), the Interferometric BIdimensional

^ Operated by the Association of Universities for Research in Astronomy, Inc. (AURA), for the National Science Foundation.
Spectrometer (IBIS, Cavallini 2006; Reardon \& Cavallini 2008), and the CRisp Imaging Spectro-Polarimeter (CRISP, Scharmer et al. 2008). These instruments differ mostly in how the FPIs are mounted (telecentric or collimated) and in the spectral resolution.

In the category of slit-based spectropolarimeters, we have the Advanced Stokes Polarimeter (ASP, Skumanich et al. 1997) which has been superseded by the the DiffractionLimited Spectro-Polarimeter (DLSP, Sankarasubramanian et al. 2003), the multiline polarimetric mode of THEMIS (MTR, Rayrole \& Mein 1993; López Ariste et al. 2000), the POlarimetric LIttrow Spectrograph (POLIS, Beck et al. 2005b), the Spectro-Polarimeter for Infrared and Optical Regions (SPINOR, Socas-Navarro et al. 2006), and the Facility InfraRed Spectropolarimeter (FIRS, Jaeggli et al. 2008). DLSP and POLIS work at a fixed wavelength in the visible $(630 \mathrm{~nm})$, while SPINOR and FIRS allow to observe different spectral regions in the visible and the infrared. The Tenerife Infrared Polarimeter (TIP, Martínez Pillet et al. 1999; Collados et al. 2007) can observe any part of the near-infrared spectrum from 1 to $2.2 \mu \mathrm{m}$ using the main spectrograph of the German Vacuum Tower 
Telescope (VTT). It regularly reaches the diffraction limit at these wavelengths (about 0.'6).

All ground-based instruments suffer from wavefront distortions caused by turbulence in the Earths' atmosphere. Adaptive optic systems (AO, von der Lühe et al. 2003; Scharmer et al. 2003; Rimmele 2004) are mandatory to improve the image quality, but the compensation is only partial and sub-arcsec resolution is hard to reach. Whereas in slit-based spectropolarimetry no further improvement can be achieved beyond what is delivered by the AO system, two-dimensional spectropolarimetric observations can benefit from post-facto image reconstruction techniques like de-stretching, speckle deconvolution (see, e.g., Keller \& von der Luehe 1992; Mikurda et al. 2006; Puschmann \& Sailer 2006; Bello González \& Kneer 2008), and MOMFBD (van Noort et al. 2005). At present, the only instrument capable of delivering continuous measurements at 0.3 resolution is the spectropolarimeter (SP) of the Hinode satellite (Kosugi et al. 2007). The Imaging Magnetograph eXperiment (IMaX, Jochum et al. 2003; Martínez Pillet et al. 2004; Álvarez-Herrero et al. 2006) aboard the SUNRISE balloon (Gandorfer et al. 2006) recently completed its first flight and took data of even higher resolution thanks to its 1-m telescope.

However, accurate spectropolarimetry does not only depend on spatial resolution, but also on the precision achieved in the determination of the polarization state. This is typically quantified by the root-mean-square (rms) noise of Stokes $Q U V$ at continuum wavelengths or by the signal-to-noise ratio (SNR) in the intensity spectrum. Slit spectropolarimeters have the advantage that the spectrum is detected instantly. Hence, an increase in exposure time or the accumulation of spectra do not compromise the spectral purity or integrity of the data. With integration times of $20-30 \mathrm{~s}$, the rms noise can be reduced to a few $10^{-4}$ of the continuum intensity (Bommier \& Molodij 2002; Khomenko et al. 2003; Martínez González et al. 2008; Beck \& Rezaei 2009). For FPI-based instruments this is more difficult due to the sequential wavelength scanning. To obtain the SNR of a typical slit-spectrograph spectrum, a 2D instrument has to use a similar integration time for every wavelength position, which usually makes the line scan unacceptably long ( $>2 \mathrm{~min})$. Hence, for all 2D-type spectropolarimeters a compromise has to be found between SNR, spectral sampling, and angular resolution. A SNR comparable to that of long-integrated ( $\gg 5 \mathrm{~s}$ ) slit-spectra cannot be obtained with any currently existing $2 \mathrm{D}$ instrument.

However, these instruments also have great advantages compared to slit systems. First, they are flexible in wavelength range. Even if the various spectral regions can be observed sequentially only, the cadence may be sufficiently high to treat them as simultaneous, depending on the scientific problem. For slit-based systems, a change of the spectral region usually requires changes in the setup, or is even impossible because of fixed gratings (DLSP, POLIS, Hinode/SP). Second, 2D instruments provide spatially coherent observations with much faster cadence than is possible with slit systems. In high-resolution mode, when small step sizes are involved (e.g., $0{ }^{\prime} 15$ in the case of the Hinode/SP or the DLSP), slit-spectrograph systems do not attain the cadence needed to follow the solar evolution with sufficient spatial coverage. Fast events, particularly in the solar chromosphere with time scales of 30 s or less (Rutten \& Uitenbroek 1991; Carlsson \& Stein 1997; Wedemeyer et al. 2004; Wöger et al. 2006; Beck et al. 2008), can only be traced on very small FOVs, whereas the spatial extent of the phenomenon may excess the scan area easily.

The Triple Etalon SOlar Spectrometer (TESOS, Kentischer et al. 1998; Tritschler et al. 2002) at the German VTT in Tenerife was a natural candidate for an upgrade into a vector polarimeter. TESOS features high spectral resolution and is the only tripleFPI system in operation to date. The unique spectroscopic capabilities of TESOS have been used to study the kinematic and thermal properties of several magnetic and non-magnetic structures (see, e.g., Schlichenmaier \& Schmidt 1999; Schmidt \& Schlichenmaier 2000; Langhans et al. 2002; Schleicher et al. 2003; Tritschler et al. 2004; Schlichenmaier et al. 2004; Bellot Rubio et al. 2006; Mikurda et al. 2006). However, although valuable for the analysis of photospheric velocity fields and temperatures, these observations do not provide information about the magnetic field.

Here we describe the KIS/IAA Visible Imaging Polarimeter (VIP), the polarization package that converts TESOS into a full vector spectropolarimeter. Section 2 gives an overview of the optical layout of TESOS in polarimetric mode, focusing on the modulation package and the detectors. The data acquisition and the calibration procedure are explained in Sects. 3 and 4, respectively. In Sect. 5, we present three examples of VIP observations to illustrate the capabilities of the instrument. Our conclusions are summarized in Sect. 6.

\section{Instrument description}

TESOS is a Fabry-Pérot spectrometer in a telecentric configuration (Kentischer et al. 1998; Tritschler et al. 2002). Figure 1 sketches the optical layout with its current components. The three etalons significantly reduce side-lobe influence and permit the use of broad prefilters $(\sim 1 \mathrm{~nm} F W H M)$ with typical transmissions of $75 \%$. In high resolution mode, the spectral resolution of the instrument is of the order of 300000 at $632.8 \mathrm{~nm}$, comparable with classical slit spectrographs. Up to four spectral lines can be observed sequentially thanks to the motorized filter wheel (IF1 in Fig. 1). Combined with the Kiepenheuer Adaptive Optics System (von der Lühe et al. 2003), TESOS is able to achieve a spatial resolution of about 0.5 on a regular basis.

Polarimetry was one of the science drivers of TESOS from the beginning (see Kentischer et al. 1998). To minimize instrumental polarization, the four folding mirrors inside TESOS were arranged in such a way that two sagittal $45^{\circ}$ reflections are followed by two tangential $45^{\circ}$ reflections. This configuration does not introduce spurious linear polarization signals and thus allows for high precision polarimetry.

The KIS/IAA VIP has been developed by the Kiepenheuer Institut für Sonnenphysik and the Instituto de Astrofísica de Andalucía to upgrade TESOS into a full vector spectropolarimeter. VIP consists of a modulation package, its electronics, a Wollaston prism, and the control software. With some technical modifications, it could also be used at the main spectrograph of the VTT.

\subsection{Modulation package and polarization analysis}

The incoming light beam is modulated by two nematic liquid crystal variable retarders (LCVRs) manufactured by Meadowlark. The LCVRs are located directly in front of the field stop in a converging beam $(f / 64)$ close to the focal plane (LCVR1 and LCVR2 in Fig. 1). A suitable blocking filter protects them from UV damage. The two LCVRs are mounted with their fast axes making an angle of $45^{\circ}$.

LCVRs are electro-optical tunable retarders made of liquid crystal molecules enclosed between two glass plates. Without an external electric field, all molecules are aligned with their 


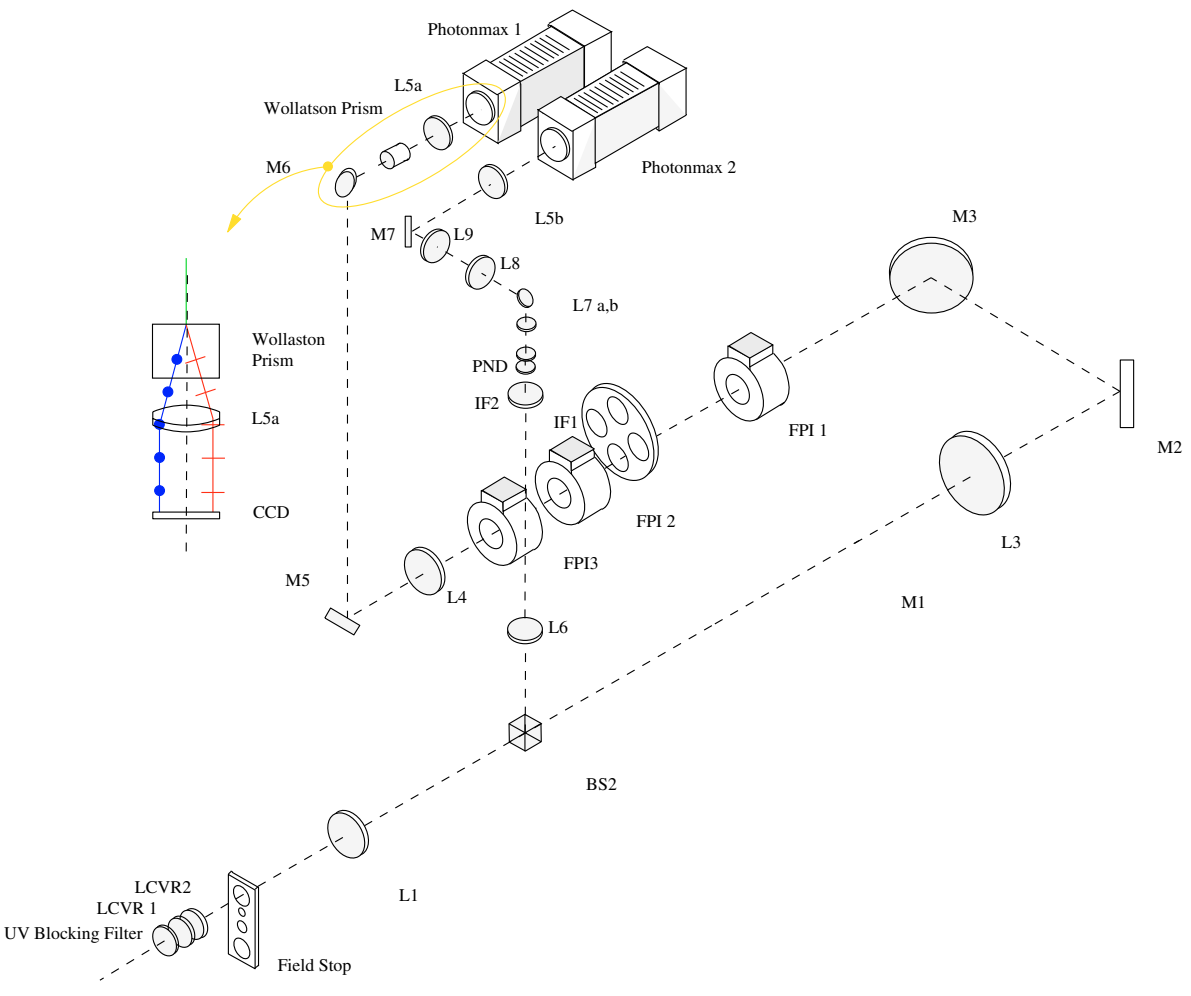

Fig. 1. Optical setup of TESOS in high resolution mode. The light undergoes four $45^{\circ}$ reflections at the mirrors M2, M3, M5 and M6. The Fabry-Pérot etalons are indicated by the abbreviation FPI. The filter wheel (IF1) is located between FPI 1 and FPI2. The modulation package consists of two LCVRs near the telescope focal plane. PhotonMax 1 and 2 are the cameras of the narrow-band and broad-band channels, respectively. The Wollaston prism in front of PhotonMax 1 performs the polarization analysis.

long axis parallel to the glass substrate and maximum retardance is achieved. If an external alternating electrical field is applied (square wave, $2 \mathrm{kHz}$ ), the molecules begin to tilt perpendicular to the glass substrate, which causes a reduction of the effective birefringence. The VIP LCVRs can be used in the range from 420 to $700 \mathrm{~nm}$; their retardance was measured as a function of the applied voltage for two wavelengths $(530 \mathrm{~nm}$ and $630 \mathrm{~nm}$, see Fig. 2). The voltage needed for a specific retardance and wavelength is then derived by a linear interpolation of the calibration curves (Kentischer 2005). At low voltages, the interaction forces within the crystals are the dominant effects. Therefore their relaxation time is longest in this regime (up to $60 \mathrm{~ms}$ ). To give the LCVRs enough time to reach the desired retardance, the modulation sequence of VIP was chosen such that these transitions occur when the instrument is busy with etalon settings, camera readout, or disk writing.

Dual-beam polarimetry is achieved using a Wollaston prism before the camera lens L5a and the detector (see Fig. 1, zoom-in area). It is oriented parallel to the fast axis of the first LCVR and acts as a polarization analyzer. The two orthogonal beams generated by the Wollaston are imaged simultaneously onto the same detector. In the data reduction process, they are combined to minimize seeing-induced crosstalk (Lites 1987). To reduce image distortions between the two beams, the Wollaston is made of calcite instead of quartz. The splitting angle of the prism, $1.37^{\circ}$, was optimized to fill the entire CCD.

\subsection{Detectors}

The PixelVision Pluto cameras used for TESOS were replaced in 2006. The new detector system consists of two 16-bit, high frame rate PhotonMax CCD cameras manufactured by Princeton Instruments/Acton. Dark current is minimized by thermo-electrical cooling down to $-80{ }^{\circ} \mathrm{C}$. The CCD image sensor is a backside illuminated, $512 \times 512$ pixel array $(\mathrm{e} 2 \mathrm{v}$ CCD97) with frame transfer technology and a square pixel size of $16 \mu \mathrm{m}$. The wide dynamic range, large full-well capacity

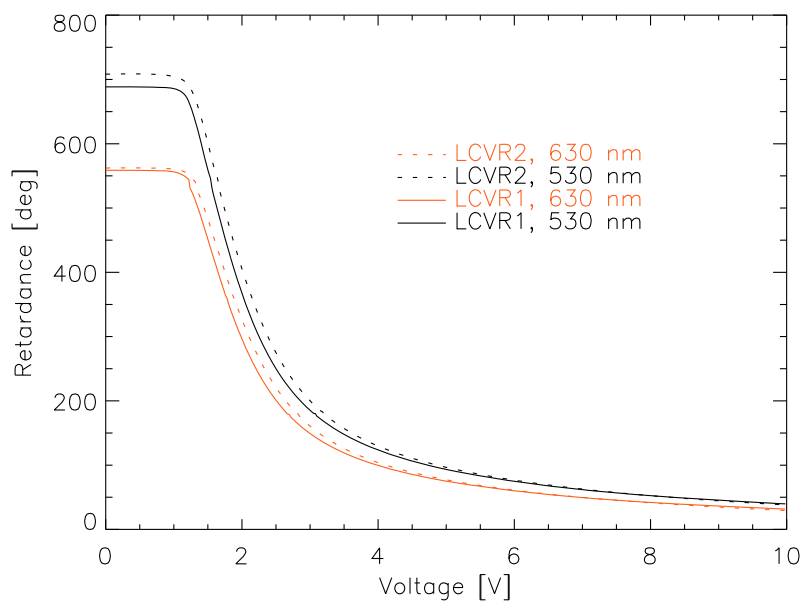

Fig. 2. Calibration curves of the two LCVRs for the reference wavelengths of $530 \mathrm{~nm}$ (black) and $630 \mathrm{~nm}$ (red). The voltage was increased in steps of $0.02 \mathrm{~V}$.

(200 $\mathrm{ke}^{-}$in traditional amplification mode) and high quantum efficiency ( $>60 \%$ in the wavelength range $400-850 \mathrm{~nm}$ ) of the cameras make them ideal for spectropolarimetry.

As a consequence of the different array and pixel size of the new detector, the imaging optics in the narrow-band channel of TESOS had to be changed in order to allow for a FOV of about $40^{\prime \prime}$ in diameter, while maintaining the pixel scale. The optimum result was achieved with a $f=169.7 \mathrm{~mm}$ lens, which provides a pixel scale of $0{ }^{\prime} 086$ pixel $^{-1}$ and a FOV of $21^{\prime \prime} \times 41^{\prime \prime}$ in spectropolarimetric mode.

\section{Spectropolarimetric data acquisition}

To determine the Stokes vector, VIP takes four images $I_{j}$ $(j=1 \ldots 4)$ with the LCVRs in different modulation states. 
Following the IMaX strategy (Martínez Pillet et al. 2004), the retardances of the first and second LCVR are set to $\left(315^{\circ}, 315^{\circ}\right.$, $\left.225^{\circ}, 225^{\circ}\right)$ and $\left(305.264^{\circ}, 54.736^{\circ}, 125.264^{\circ}, 234.736^{\circ}\right)$, respectively. This results in four linearly independent combinations

$$
\left(\begin{array}{l}
I_{1} \\
I_{2} \\
I_{3} \\
I_{4}
\end{array}\right)=\left(\begin{array}{cccc}
1 & 1 / \sqrt{3} & 1 / \sqrt{3} & 1 / \sqrt{3} \\
1 & 1 / \sqrt{3} & -1 / \sqrt{3} & -1 / \sqrt{3} \\
1 & -1 / \sqrt{3} & -1 / \sqrt{3} & 1 / \sqrt{3} \\
1 & -1 / \sqrt{3} & 1 / \sqrt{3} & -1 / \sqrt{3}
\end{array}\right) \cdot\left(\begin{array}{c}
I \\
Q \\
U \\
V
\end{array}\right),
$$

from which the Stokes $I, Q, U$ and $V$ parameters at the position of the LCVRs can be derived.

The theoretical efficiencies are $\left(\epsilon_{I}, \epsilon_{Q}, \epsilon_{U}, \epsilon_{V}\right)=$ $(1,1 / \sqrt{3}, 1 / \sqrt{3}, 1 / \sqrt{3})$, with $1 / \sqrt{3} \sim 0.577$. This provides the best modulation possible with equal efficiency in all Stokes parameters (del Toro Iniesta \& Collados 2000). The polarimeter can also be used to measure only Stokes $I$ and $V$; the retardances of the LCVRs are then $\left(360^{\circ}, 360^{\circ}\right)$ and $\left(90^{\circ}, 270^{\circ}\right)$, respectively. To improve the SNR, multiple images for each modulation state can be accumulated by the control software. Up to now, we have used this option only to obtain flat field data, since the extra read-out time increases significantly the duration of the wavelength scans; for observations, we use long exposures and the large full-well capacity of the CCDs to reach a sufficiently high SNR.

\section{Data calibration}

For the most part, the data reduction process is identical to the one performed when the instrument is operated in spectroscopic mode, which is described in detail elsewhere (see, e.g., Tritschler et al. 2004). The reduction provides flat-fielded and aligned images for each wavelength, modulation state, and orthogonal beam. The alignment of the different scan steps is done using the simultaneous images acquired with the broad-band camera of TESOS (PhotonMax 2 in Fig. 1) as a reference. Here, we only focus on the aspects that are special to spectropolarimetry.

The general approach for the polarimetric calibration of VIP is similar to that of the other spectropolarimeters at the VTT (POLIS, TIP, GFPI). The time-dependent instrumental polarization introduced by the telescope and the remaining optics behind the exit window of the telescope is corrected separately in two steps as described in detail by Beck et al. (2005a), and Schlichenmaier \& Collados (2002) or Beck et al. (2005b); for the GFPI see Bello González \& Kneer (2008).

To determine the response function, $X$, of the polarimeter and the optics behind the instrument calibration unit (ICU), 37 known polarization states are created by the ICU and measured with VIP (see Fig. 3). The ICU is located right behind the exit window of the evacuated telescope, and consists of a linear polarizer and a zero-order quartz retarder. The retarder is rotated in $5^{\circ}$ steps from $0^{\circ}$ to $180^{\circ}$, with the transmission axis of the polarizer fixed along the terrestrial N-S direction. Calibration curves for each of the LCVR states are obtained from the intensity difference between the two orthogonal beams produced by the Wollaston, normalized to their average value (Fig. 3). A flat field correction is not needed for the calibration measurements, because gain table variations affect the difference and average images by exactly the same multiplicative factor, and thus they cancel out. Tests with and without flat field correction yielded the same polarimetric response down to our accuracy level. The subtraction of the dark current is, however, crucial because it influences the relative measurements of intensities in a non-linear
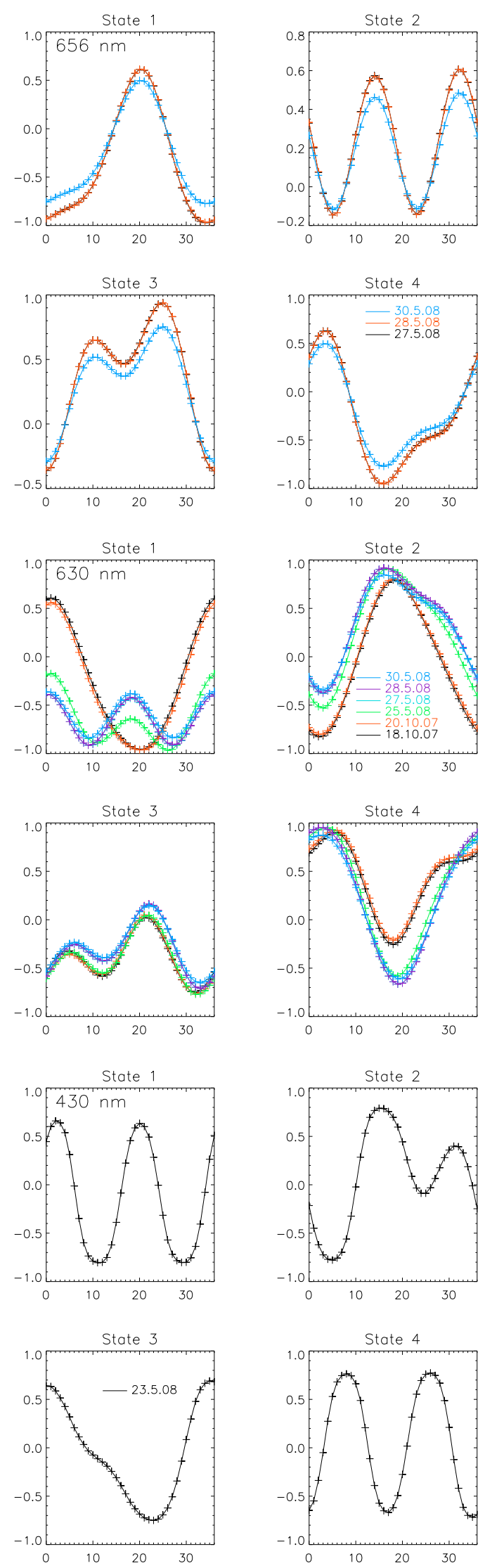

Fig. 3. Calibration curves for the four modulation states at 656, 630, and $430 \mathrm{~nm}$ (from top to bottom). Calibrations belonging to different days are given in different colors; in some cases the curves are nearly identical and cannot be distinguished from each other (e.g., black and red curve in top four panels). 
C. Beck et al.: Solar spectropolarimetry with the visible imaging polarimeter

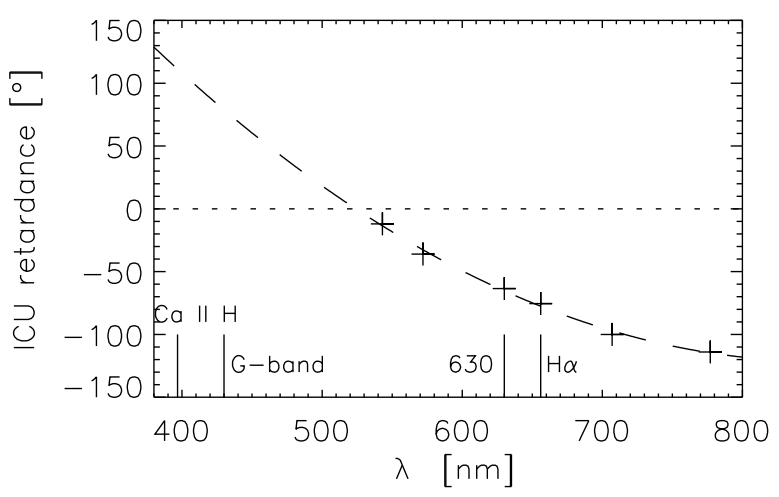

Fig. 4. Retardance of the visible ICU at the VTT. Crosses denote measurements, the dashed line a parabolic fit.

way. The four calibration curves can then be used to determine $X$ by a matrix inversion as described in Appendix A. 2 of Beck et al. (2005b). Applied to the four intensity measurements that result from subtracting the two orthogonal beams for each LCVR state, $X$ gives the Stokes parameters at the position of the ICU.

VIP differs from slit spectropolarimeters in its 2D FOV, which implies that also the polarization properties can vary in both spatial dimensions. We investigated the derived response functions for subfields of the VIP FOV, but found no significant spatial variation within the accuracy of $X$. Therefore we use the same response function for every CCD pixel. We also found no significant trend with wavelength inside the typical range used to scan a line, so we compute $X$ only for the first wavelength position (usually a continuum point).

TESOS, and hence VIP, is prepared for sequential multiwavelength observations. This means that the calibration has to be done for as many spectral regions as used in the observations, but a calibration data set can be taken in less than 15 minutes. The variation of the ICU retardance with wavelength is shown in Fig. 4. The parameters of a parabolic fit to the measured retardances are $(688,-1.89537,0.00110836)$ for the offset, slope, and 2 nd order contribution. The chromatic wave plate of the ICU has zero retardance around $520 \mathrm{~nm}$ and cannot be used in this spectral region.

A critical issue for the accuracy of the measurements is the stability of the polarization modulation over time. We consider here the nematic LCVRs as the major source of variation, since the remainder of the setup consists of "stable" massive objects like mirrors, the FPIs, the Wollaston, or beam splitters (BS) that feed additional imaging channels in front of TESOS. The LCVRs can change the polarimetric response of the instrument since their effective retardance is temperature dependent. The retardance decreases by about 1 degree for an increase in temperature by $1 \mathrm{~K}$ (see, e.g., Heredero et al. 2007, their Fig. 5). Measurements at the ICU retarder showed a temperature increase of about $0.6^{\circ}$ in two minutes on being exposed to sunlight before the temperature leveled off, as well as a reduction of retardance by $0.4^{\circ}$ at $630 \mathrm{~nm}$ comparing measurements in the early morning and at noon (Beck 2004). The light absorption of the LCVRs, and hence, their behavior, should be similar. During the observations, the LCVRs are permanently exposed to the sunlight, since no shutters or similar devices are located in front of them. There should thus only be some minor and fast heating effect before the start of the first observation in the early morning
Table 1. Overview of the observations. $I_{\mathrm{c}}$ : continuum intensity.

\begin{tabular}{lccc}
\hline \hline Date & Nov. 3, 2005 & Oct. 18, 2007 & May 23, 2008 \\
\hline Target & Pore & Network & Network \\
Position & $10^{\circ}$ & $20^{\circ}$ & $30^{\circ}$ \\
VIP FOV & $26^{\prime \prime} \times 35^{\prime \prime}$ & $18^{\prime \prime} \times 40^{\prime \prime}$ & $16^{\prime \prime} \times 38^{\prime \prime}$ \\
Wavelength & $630.2 \mathrm{~nm}$ & $630.25 \mathrm{~nm}$ & $430.34 \mathrm{~nm}$ \\
Line(s) & $2 \mathrm{Fe} \mathrm{I}, \mathrm{O}_{2}$ & $\mathrm{Fe}, \mathrm{O}_{2}$ & $\mathrm{CH}, \mathrm{Fe}$ II \\
Step Size & $1.9 \mathrm{pm}$ & $1.9 \mathrm{pm}$ & $1.6 \mathrm{pm}$ \\
Points & $42+46$ & 35 & 68 \\
Exposure & $300 \mathrm{~ms}$ & $300 \mathrm{~ms}$ & $300 \mathrm{~ms}$ \\
Cadence & $72 \mathrm{~s}+79 \mathrm{~s}$ & $70 \mathrm{~s}$ & $120 \mathrm{~s}$ \\
Noise level & $2 \times 10^{-3} I_{\mathrm{c}}$ & $2 \times 10^{-3} I_{\mathrm{c}}$ & $3 \times 10^{-3} I_{\mathrm{c}}$ \\
TIP FOV & - & $4.8^{\prime \prime} \times 78^{\prime \prime}$ & $12.5^{\prime \prime} \times 78^{\prime \prime}$ \\
Imaging & - & $\mathrm{G}-\mathrm{band}$ & $\mathrm{G}-\mathrm{band}$ \\
\hline
\end{tabular}

Notes. ${ }^{1}$ Including disk write time; the scan time is about $10-20 \mathrm{~s}$ shorter, depending on the number of wavelength points.

and a slight drift with time caused by the change of the light level during the day.

The LVCRs of VIP are not located in a temperaturecontrolled housing, but the observing room as a whole is airconditioned with a nominal temperature of $21^{\circ} \mathrm{C}$. Temperature changes of the surrounding air will presumably not exceed a level of one degree due to the air-condition. Taking one degree of temperature and thus one degree of retardance as the uppermost limit of variation, this translates into a deviation from the default modulation scheme by about three percent (e.g., $\left.\cos 304^{\circ} / \cos 305^{\circ}=0.975\right)$. This three percent is, however, not an absolute but a relative error; for instance for a polarization signal with an amplitude of $10 \%$ of the continuum intensity, the resulting error would be only $0.3 \%$. Such an error level is comparable to the rms noise of the data (see Table 1). The value of the rms noise actually implies that any short-term variations of temperature on the order of a few seconds cannot reach one degree, since it provides an upper limit for the contributions of all noise sources in addition to the thermal retardance effects. The main effect of the temperature changes will thus be a slow drift of retardance with time that presumably can be neglected for the polarimetric accuracy if the calibration is done right before or after the observations.

In order to verify the stability of the calibration, we collected calibration curves from several campaigns for wavelengths of $430 \mathrm{~nm}, 630 \mathrm{~nm}$, and $656 \mathrm{~nm}$ (Fig. 3). The curves demonstrate that the calibration is stable to within the measurement accuracy for up to two-three days (e.g., $630 \mathrm{~nm}$ on October 18 and 20, 2007 or $656 \mathrm{~nm}$ on May 27 and 28, 2008). The large changes in the shape of the curves (compare 2007 (red) and 2008 (blue) in state $1,630 \mathrm{~nm}$ ) are due to the additional BS used in 2008 to feed the imaging channels; the minor changes between, e.g., May 25 and 30 were due to a re-adjustment of the same BS. We conclude that daily calibration measurements should suffice to maintain a high polarimetric accuracy for VIP if the optical setup remains unchanged.

The measured polarization efficiencies are typically $\left(\epsilon_{I}, \epsilon_{Q}, \epsilon_{U}, \epsilon_{V}\right)=(0.96,0.53,0.52,0.56)$ at $630 \mathrm{~nm}$, slightly below the theoretical values but still very high. The instrumental polarization caused by the VTT coelostat is removed using the telescope model of Beck et al. (2005a). If the polarization level in a continuum window inside the observed spectral range differs from zero, a correction for residual $I \rightarrow Q U V$ crosstalk is applied by calculating the average $Q U V / I$ ratio in the continuum window and subtracting the corresponding 


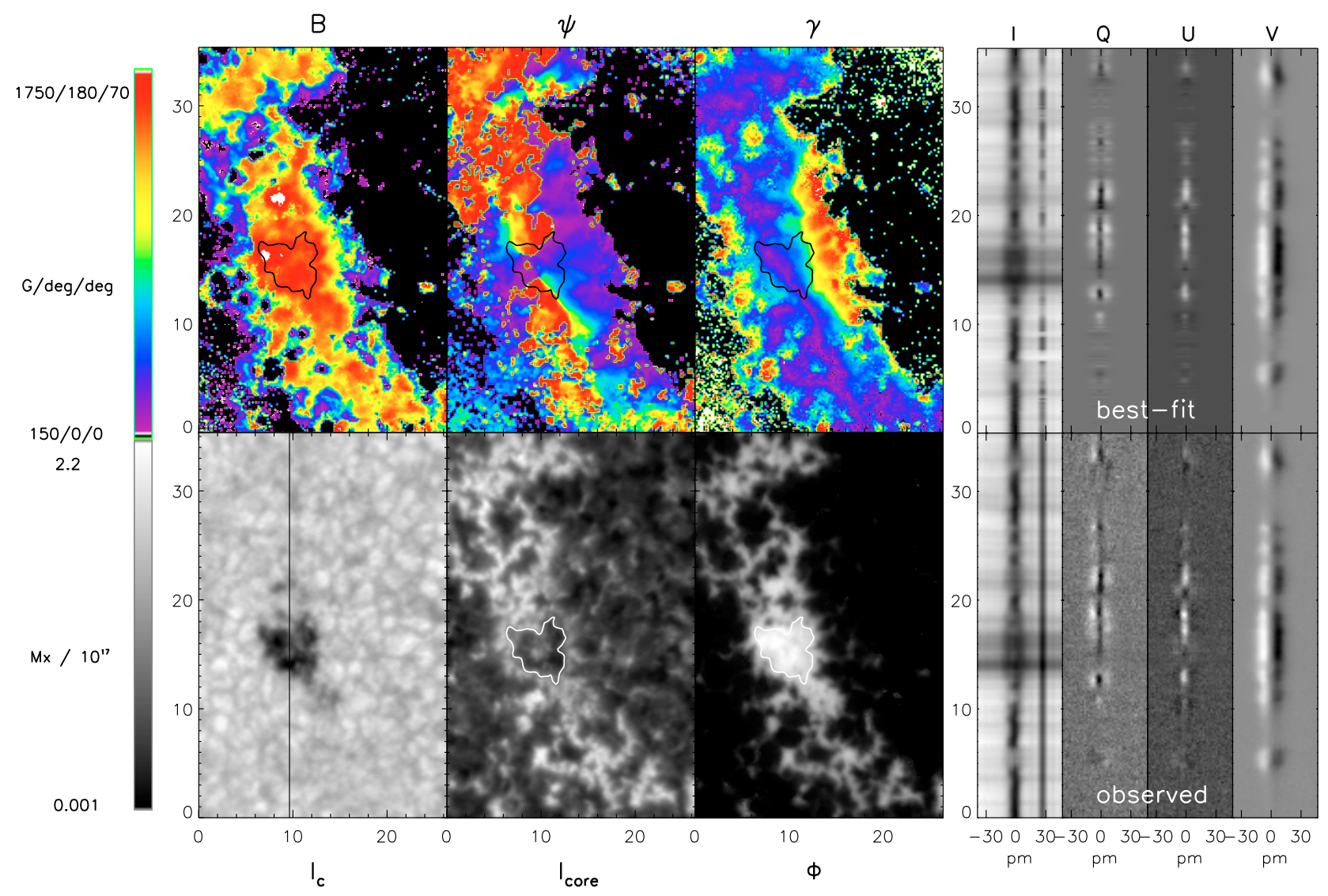

Fig. 5. Left panel: pore data taken in 2005. Bottom row, left to right: continuum intensity $I_{\mathrm{c}}$, line-core intensity of Fe I $630.25 \mathrm{~nm}$, and longitudinal magnetic flux $\Phi$. Top row, left to right: field strength $B$, field azimuth $\psi$, and field inclination $\gamma$ in the LOS reference frame. The black/white contours outline the extension of the pore. The black vertical line in $I_{\mathrm{c}}$ marks the cut whose spectra are shown in the right panels. The observed and best-fit spectra are shown at bottom and at top, respectively. Left to right: Stokes IQUV. Spatial tick marks here and in all following figures are in arcsec.

fraction of the intensity profile from $Q U V$. This crosstalk comes from two sources of calibration inaccuracies: the limitations of the geometrical telescope model and the high sensitivity of the first column of the response matrix to the retardance adopted for the ICU (see Fig. 4 of Beck et al. 2005b). The latter quickly leads to deviations of some percent (roughly $1 \%$ of crosstalk per $1^{\circ}$ error in retardance). The determination of the ICU retardance can be improved with additional calibration data at different polarizer positions as described by Beck et al. (2005b).

\section{Observations}

After commissioning, VIP has been used in several campaigns from 2005 until 2009. During the first test runs, VIP was a standalone instrument devoted to the observation of the pair of Fe I lines at $630 \mathrm{~nm}$. In later campaigns it has been operated simultaneously with TIP and additional imaging channels as, for example, the G-band at $430.5 \mathrm{~nm}$ and $\mathrm{H} \alpha$ (see, e.g., Kučera et al. 2008). In the following we describe and discuss three specific observations with VIP to demonstrate its scientific potential. Details of the observations are given in Table 1.

When coordinated with TIP, the spectrograph slit (of length $70^{\prime \prime}-80^{\prime \prime}$ ) was oriented parallel to the long axis of the VIP FOV, covering it completely. In the direction perpendicular to the slit the coverage depends on the region scanned with TIP. The setup for multi-instrument observations including VIP is described in Beck et al. (2007) and Kučera et al. (2008).
All observations are dark subtracted, flat fielded, and corrected for transparency fluctuations during the scan. For the two data sets of network no de-stretch prior to the polarization calibration has been performed. Usually the observations are binned by a factor of two to increase the signal-to-noise ratio, so the effective pixel size is 0 .' 17 .

\subsection{Pore observations}

On November 3, 2005 we observed a pore (NOAA 10818) close to disk center. VIP was used to scan the two Fe I $630.2 \mathrm{~nm}$ lines sequentially with a total of 88 wavelength points and a scan time of about $65 \mathrm{~s}$ for each line. For the following, we only considered the line at $630.25 \mathrm{~nm}$. In order to determine reliable magnetic parameters and to assess the performance of VIP, the Stokes $I, Q, U$ and $V$ profiles were inverted using the SIR code. We performed a 1-component inversion with a variable stray light contribution $\alpha$ fitted by the algorithm. Depending on whether the polarization signal exceeded a threshold of $0.85 \%$ of the continuum intensity or not, either a single magnetic or a field-free atmosphere was assumed.

Figure 5 displays the observed FOV (bottom panels) and some of the inversion results (top panels). The continuum intensity (bottom left) shows clear signs of abnormal granulation surrounding the pore. The line-core intensity image (bottom middle) reveals brightenings that closely follow the course of the intergranular lanes throughout most of the FOV. The spatial 

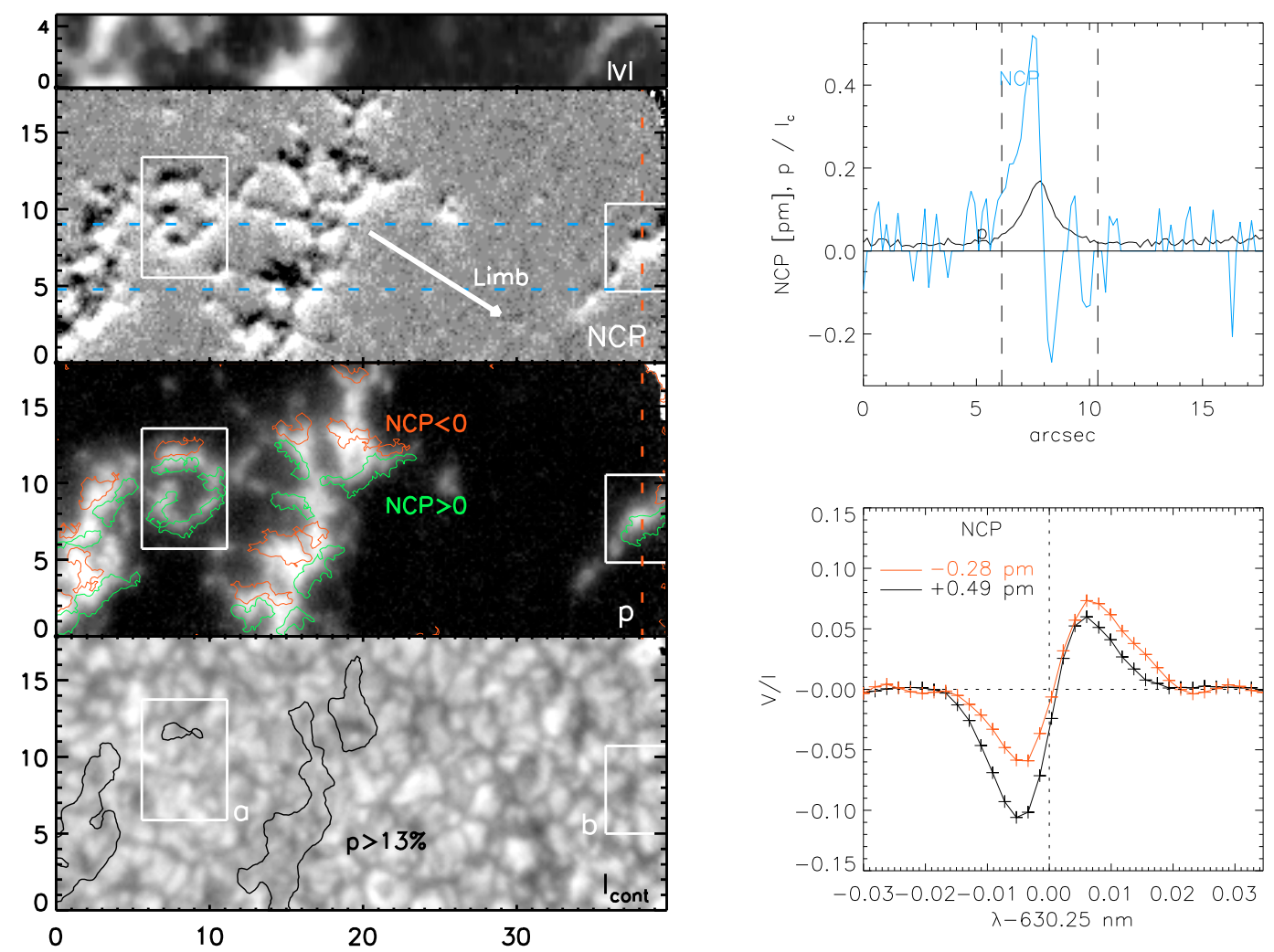

Fig. 6. Left panel: network data taken in 2007. Bottom to top: $I_{\mathrm{c}}$, polarization degree $p, \mathrm{NCP},|V|$ from TIP. Red and green contours in $p$ outline negative and positive NCP, respectively; black contours in $I_{\mathrm{c}}$ a polarization degree above $13 \%$. The blue horizontal dashed lines in NCP represent the TIP FOV. The direction to the limb is indicated by the white arrow. Right top: NCP (blue) and $p$ (black) along the cut marked by a vertical red dashed line crossing the right white rectangle, $b$. The black vertical dashed lines denote the lower and upper boundary of rectangle b. Right bottom: Stokes $V$ profiles with maximum positive and negative NCPs in the cut.

resolution is sufficient to distinguish the ribbon-like appearance of these structures, discovered by Berger et al. (2004) in G-band filtergrams taken at the Swedish 1-m Solar Telescope. A comparison with the unsigned magnetic flux $\Phi=|(1-\alpha) \cdot B \cdot \cos \gamma|($ bottom right) demonstrates that all the strong line-core brightenings are related to the presence of magnetic fields. This does not immediately imply that the upper layers of magnetic elements are hotter than their surroundings, as not only the temperature but also the Zeeman splitting or the shift of the optical depth scale in the presence of magnetic fields increase the line-core intensity. The flux concentrations reside predominantly in the intergranular lanes. The field strength (top left) ranges from $50 \mathrm{G}$ to about $2 \mathrm{kG}$. The field azimuth (top middle) and field inclination (top right) indicate radially oriented magnetic fields that extend well beyond the visible border of the pore (white/black contours). The inclination increases with radial distance from the center of the pore, reaching a maximum of about $70^{\circ}$ to the LOS. As the pore was located close to disk center, the corresponding orientation in the local reference frame (i.e., with respect to the local surface normal) will not differ much from the LOS values. In the right half of Fig. 5, the observed and best-fit spectra along a cut through the pore are shown for comparison.

We conclude that because of the fine spectral sampling and the high SNR achieved, the spectra can be well analyzed with the SIR code, allowing for an accurate determination of the magnetic field parameters at high spatial resolution. A preliminary investigation of the effect of the transmission profile of TESOS on the observed spectra, mainly using the telluric oxygen line, suggested systematic variations of line widths or line positions across the FOV of $0.1-0.2 \mathrm{pm}$ (see also von der Lühe \& Kentischer 2000; Tritschler et al. 2002; Martínez Pillet et al. 2004; Scharmer 2006; Puschmann et al. 2006; Reardon \& Cavallini 2008). This translates into an error in the field strength (velocity) of $50 \mathrm{G}\left(50 \mathrm{~ms}^{-1}\right)$ at $630 \mathrm{~nm}$, which is comparable to the error in the determination of the velocity or the magnetic field strength (see, e.g., Beck 2006) and far below their intrinsic variation across the solar surface. A more detailed discussion of the instrumental effects on the retrieved physical parameters is postponed to a future publication.

\subsection{Network observations}

The network observations taken on October 18, 2007 at about 10:00 UT were coordinated with TIP. A broad-band G-band speckle channel was added in front of TESOS to provide context information with high spatial resolution. TIP was set to repeatedly scan a small region of $\sim 5^{\prime \prime}$ near the centre of the VIP FOV $^{1}$.

Figure 6 summarizes the observations: the continuum intensity $I_{\mathrm{c}}$ (bottom left), the polarization degree $p$ (middle left), and the net circular polarization (NCP; top left) in the Fe I line at $630.25 \mathrm{~nm}$. The NCP was computed as the integral of Stokes $V$ over wavelength, multiplied by the polarity of the field. The polarization degree was determined as

$p=\max \left(\sqrt{Q^{2}(\lambda)+U^{2}(\lambda)+V^{2}(\lambda)}\right) / I_{\mathrm{QS}}$,

\footnotetext{
${ }^{1}$ See the TIP archive for an overview of the TIP data (KIS home page $\rightarrow$ Observatories $\rightarrow$ Data archives).
} 
A\&A 520, A115 (2010)

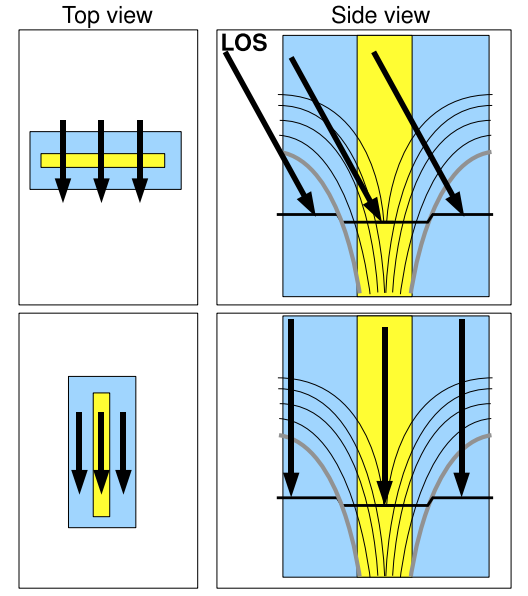

Fig. 7. Dependence of NCP behavior on the orientation of magnetic flux sheets. For an orientation of the sheet parallel to the limb (top row), the inclined LOS passes through both the central part (yellow) and the canopy (blue), for an orientation perpendicular to the limb (bottom row), it passes through either the canopy or the central axis. The LOS is inclined out of the paper plane in all but the upper right panel.

with $I_{\mathrm{QS}}$ representing the quiet Sun continuum intensity. The small map at the very top left shows the integrated $V$ signal of one TIP scan. Its location inside the VIP FOV is indicated by the blue horizontal dashed lines in the NCP map.

The NCP map reveals a distinct pattern, varying between positive and negative values on small spatial scales (e.g., left white rectangle, a). Negative NCP (black) appears preferentially toward the disk center and positive NCP (white) toward the limb. The polarization degree map shows that the highest NCP values are not cospatial with the highest polarization degree, but rather flank it. The effect is visualized in the right panel that shows $\mathrm{NCP}$ and $p$ values on a cut along the y-axis through the right white rectangle, $\mathrm{b}$. The NCP reduces to zero and changes sign across the maximum of the polarization degree. The Stokes $V$ profiles corresponding to maximal positive and negative NCP in the cut are shown at the bottom right; the pronounced asymmetry of the red and blue $V$ lobe is clearly visible in the two cases. The spectral sampling is indicated by the crosses.

Figure 7 offers a tentative explanation of how the peculiar NCP pattern could possibly be created. In the case of a flux sheet that is parallel to the limb, the LOS crosses both the canopy and the center of the sheet (top row). If the flux concentration is perpendicular to the limb (bottom row), the LOS passes through either the canopy or the central axis. This scenario provides fairly different gradients of magnetic field strength along the LOS. In addition, velocity gradients will presumably be encountered by the LOS at the boundary of the flux concentration; the field-free convective region below the canopy and the magnetic volume are expected to harbor upflows and downflows, respectively. With these velocity gradients, the two necessary conditions for generating a NCP are present (see, e.g., Auer \& Heasley 1978; Sánchez Almeida \& Lites 1992; Landolfi \& Landi Degl'Innocenti 1996). The scenario, however, will have to be investigated in more detail before it can be taken for plausible. The detection of the variation of the NCP across magnetic flux concentrations has been possible thanks to the higher spatial resolution of VIP (or Hinode, Rezaei et al. 2007) compared to older data where the resolution was not sufficient to separate the canopy from the central axis (see, e.g., Sigwarth 2001). Interestingly, a variation of the Stokes $V$ area asymmetry across magnetic elements was inferred from the inversion of ASP measurements at 1 " (Bellot Rubio et al. 2000).

\subsection{Network observations in the G band}

VIP was used for spectropolarimetry in the G band on May 23, 2008. The wavelength range covered several $\mathrm{CH}$ lines and one strong atomic line near $430.32 \mathrm{~nm}$. The short wavelength of the observations led to a rather low average intensity of about 490 counts, but the rms noise in the polarization signal stayed at about $0.3 \%$. Again, VIP was operated in coordination with TIP and an external speckle G-band channel ${ }^{2}$.

Figure 8 shows an overview of the VIP and TIP observations of a network region located at $(x, y)=\left(-446^{\prime \prime},-193^{\prime \prime}\right)$, i.e., $30^{\circ}$ away from the disk center. In this case, the two instruments covered a similar FOV. The "continuum" intensity for VIP was taken at $430.33 \mathrm{~nm}$, where the intensity in the observed wavelength range is maximum. VIP did not reveal clear Stokes $Q$ and $U$ signals above the noise level, in contrast to TIP. This may partly be attributed to the different type of lines observed, the different spectral regions, or the different SNRs achieved. The $V$ signals of both instruments, however, match closely.

The Stokes profiles recorded by VIP can be used for quantitative analyses even at the low light level prevailing in the blue. The right panel of Fig. 8 shows sample spectra from a relatively strong flux concentration marked with a red cross in the Stokes $V$ map of VIP. Besides studying how the depth of the various $\mathrm{CH}$ lines in Stokes $I$ depends on the physical properties of the plasma and the magnetic field, the observed Stokes $V$ profiles (bottom right) can be used to verify theoretical models of $\mathrm{CH}$ line formation. The overplotted blue line comes from a numerical calculation by Uitenbroek et al. (2004), for a height-independent field strength of $1 \mathrm{kG}$. The $V$ signal was scaled down by a factor of 1.5 to better reproduce the observed $\mathrm{CH}$ lines redward of $430.36 \mathrm{~nm}$. The large mismatch between observed and synthetic profiles around $430.31 \mathrm{~nm}$ is presumably caused by the parameters used to synthesize the atomic line of Fe II (A. Asensio Ramos, priv. comm.).

\section{Summary}

The KIS/IAA Visible Imaging Polarimeter (VIP) is a new instrument for 2D spectropolarimetry of the solar atmosphere. It is used with TESOS, the triple etalon spectrometer installed at the German Vacuum Tower Telescope. The polarimeter is based on a pair of nematic liquid crystal retarders and a Wollaston prism. In combination with the adaptive optics system of the telescope, VIP and TESOS provide full Stokes vector measurements of spectral lines in the visible at a resolution of 0.5 or better. Using exposure times of $300 \mathrm{~ms}$ for each modulation state and $2 \times 2$ binning, the noise level is about $0.2 \%$ of the continuum intensity. The four Stokes profiles can be measured at 40 wavelength positions in about $60 \mathrm{~s}$. The response function of the polarimeter is determined using the instrument calibration unit of the telescope, and turns out to be stable over a few days.

The high resolving power and excellent performance of TESOS and VIP make it possible to derive the magnetic field geometry and obtain information about the atmospheric conditions by means of inversion techniques. The Stokes spectra

\footnotetext{
2 In this observation, the spectral resolution of TESOS was lower than usual because of a mistake in the setup (the parallelism of the FPIs was not adjusted).
} 

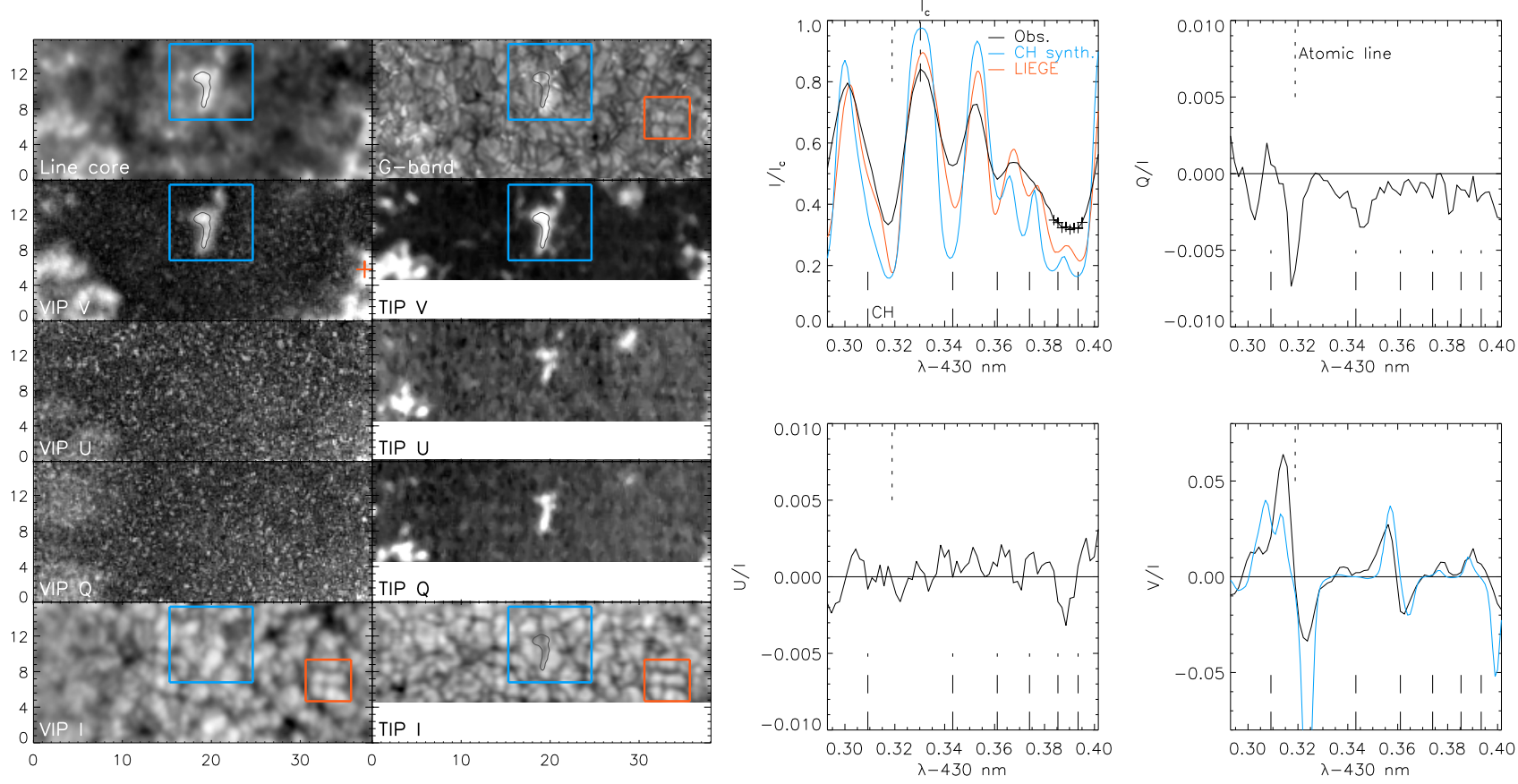

Fig. 8. Left panel: 2008 G-band observations. Left column, bottom to top: Stokes I and wavelength-integrated absolute $Q U V$, and line-core intensity near $430.39 \mathrm{~nm}$, as measured by VIP. Right column, bottom to top: Stokes $I$ and wavelength-integrated absolute $Q U V$ signals from TIP, and speckle-reconstructed broad-band G-band image. The blue and red rectangles identify the features used for the data alignment. The black contours outline large $V$ signals in TIP. The red cross in the circular polarization map from VIP near $\left(40^{\prime \prime}, 7^{\prime \prime}\right)$ marks the location of the Stokes IQUV spectra shown in the right panel. Black: profiles observed with VIP; red: Liège atlas profiles; blue: G-band synthesis (courtesy of A. Asensio Ramos, IAC). CH lines are marked with dashed vertical lines from bottom up, one atomic line with a vertical dotted line from top down. In Stokes $I$, a short bar at $430.33 \mathrm{~nm}$ denotes the continuum wavelength, and the black crosses around $430.39 \mathrm{~nm}$ the line-core intensity wavelength range.

recorded by VIP show strong asymmetries, as expected for highresolution measurements. Thus, it should be possible to determine vertical gradients of the atmospheric parameters from them. Photospheric and chromospheric lines (e.g., $\mathrm{H} \alpha$ ) can be observed sequentially thanks to the motorized filter wheel of TESOS.

Usually VIP is operated in coordination with the Tenerife Infrared Polarimeter and speckle imaging systems at the German Vacuum Tower Telescope, providing multi-wavelength observations of the same structures and processes. VIP could also be used for coordinated observations with space-borne instruments such as Hinode or the upcoming Solar Dynamic Observatory. Because of these reasons, we anticipate that future observations with VIP will be used to address several open questions in solar physics.

Acknowledgements. The VTT is operated by the Kiepenheuer-Institut für Sonnenphysik, Freiburg, Germany, at the Spanish Observatorio del Teide of the Instituto de Astrofísica de Canarias. This work has been partially funded by the Spanish Ministerio de Ciencia e Innovación through project ESP2006-13030C06-02 (including European FEDER funds) and through project AYA 200763881 .

\section{References}

Álvarez-Herrero, A., Belenguer, T., Pastor, C., et al. 2006, in SPIE Conf. Ser. 6265, ed. J. C. Mather, H. A. MacEwen, \& M. W. M. de Graauw, 132 Auer, L. H., \& Heasley, J. N. 1978, A\&A, 64, 67

Beck, C. 2004, Technical note, Kiepenheuer Institut für Sonnenphysik, Freiburg Beck, C. 2006, Ph.D. Thesis, Albert-Ludwigs-University, Freiburg

Beck, C., \& Rezaei, R. 2009, A\&A, 502, 969

Beck, C., Schlichenmaier, R., Collados, M., Bellot Rubio, L., \& Kentischer, T. 2005a, A\&A, 443, 1047

Beck, C., Schmidt, W., Kentischer, T., \& Elmore, D. 2005b, A\&A, 437, 1159
Beck, C., Mikurda, K., Bellot Rubio, L. R., Kentischer, T., \& Collados, M. 2007, in Modern solar facilities - advanced solar science, ed. F. Kneer, K. G. Puschmann, \& A. D. Wittmann, 55

Beck, C., Schmidt, W., Rezaei, R., \& Rammacher, W. 2008, A\&A, 479, 213

Bello González, N., \& Kneer, F. 2008, A\&A, 480, 265

Bellot Rubio, L. R., Ruiz Cobo, B., \& Collados, M. 2000, ApJ, 535, 489

Bellot Rubio, L. R., Schlichenmaier, R., \& Tritschler, A. 2006, A\&A, 453, 1117

Berger, T. E., Rouppe van der Voort, L. H. M., Löfdahl, M. G., et al. 2004, A\&A, 428,613

Bommier, V., \& Molodij, G. 2002, A\&A, 381, 241

Carlsson, M., \& Stein, R. F. 1997, ApJ, 481, 500

Cavallini, F. 2006, Sol. Phys., 236, 415

Collados, M., Lagg, A., Díaz García, J. J., et al. 2007, in The Physics of Chromospheric Plasmas, ed. P. Heinzel, I. Dorotovič, \& R. J. Rutten, ASP Conf. Ser., 368, 611

del Toro Iniesta, J. C., \& Collados, M. 2000, Appl. Opt., 39, 1637

Gandorfer, A. M., Solanki, S. K., Barthol, P., et al. 2006, in SPIE Conf. Ser., ed. L. M. Stepp, 6267, 25

Heredero, R. L., Uribe-Patarroyo, N., Belenguer, T., et al. 2007, Appl. Opt., 46, 689

Jaeggli, S. A., Lin, H., Mickey, D. L., et al. 2008, AGU Spring Meeting Abstracts, A11

Jochum, L., Collados, M., Martínez Pillet, V., et al. 2003, in SPIE Conf. Ser., ed. S. Fineschi, 4843, 20

Keller, C. U., \& von der Luehe, O. 1992, A\&A, 261, 321

Kentischer, T. 2005, Technical note, Kiepenheuer Institut für Sonnenphysik, Freiburg

Kentischer, T. J., Schmidt, W., Sigwarth, M., \& von Uexküll, M. 1998, A\&A, 340,569

Khomenko, E. V., Collados, M., Solanki, S. K., Lagg, A., \& Trujillo Bueno, J. 2003, A\&A, 408, 1115

Kosugi, T., Matsuzaki, K., Sakao, T., et al. 2007, Sol. Phys., 243, 3

Kučera, A., Beck, C., Gomory, P., et al. 2008, 12th European Solar Physics Meeting, Freiburg, Germany, held September 8-12, http://espm.kis. uni-freiburg.de/, 12, 2

Landolfi, M., \& Landi Degl'Innocenti, E. 1996, Sol. Phys., 164, 191

Langhans, K., Schmidt, W., \& Tritschler, A. 2002, A\&A, 394, 1069

Lites, B. W. 1987, Appl. Opt., 26, 3838

López Ariste, A., Rayrole, J., \& Semel, M. 2000, A\&AS, 142, 137 
Martínez González, M. J., Collados, M., Ruiz Cobo, B., \& Beck, C. 2008, A\&A, 477, 953

Martínez Pillet, V., Collados, M., Sánchez Almeida, J., et al. 1999, in ASP Conf. Ser., 183, 264

Martínez Pillet, V., Bonet, J. A., Collados, M. V., et al. 2004, in SPIE Conf. Ser. 5487, ed. J. C. Mather, 1152

Mickey, D. L., Canfield, R. C., Labonte, B. J., et al. 1996, Sol. Phys., 168, 229

Mikurda, K., Tritschler, A., \& Schmidt, W. 2006, A\&A, 454, 359

Puschmann, K. G., \& Sailer, M. 2006, A\&A, 454, 1011

Puschmann, K. G., Kneer, F., Seelemann, T., \& Wittmann, A. D. 2006, A\&A, 451, 1151

Rayrole, J., \& Mein, P. 1993, in The Magnetic and Velocity Fields of Solar Active Regions, ed. H. Zirin, G. Ai, \& H. Wang, IAU Colloq., 141, ASP Conf. Ser., 46, 170

Reardon, K. P., \& Cavallini, F. 2008, A\&A, 481, 897

Rezaei, R., Steiner, O., Wedemeyer-Böhm, S., et al. 2007, A\&A, 476, L33

Rimmele, T. R. 2004, in SPIE Conf. Ser. 5490, ed. D. Bonaccini Calia, B. L. Ellerbroek, \& R. Ragazzoni, 34

Rutten, R. J., \& Uitenbroek, H. 1991, Sol. Phys., 134, 15

Sánchez Almeida, J., \& Lites, B. W. 1992, ApJ, 398, 359

Sankarasubramanian, K., Elmore, D. F., Lites, B. W., et al. 2003, in SPIE Conf. Ser. 4843, ed. S. Fineschi, 414

Scharmer, G. B. 2006, A\&A, 447, 1111

Scharmer, G. B., Dettori, P. M., Lofdahl, M. G., \& Shand, M. 2003, in SPIE Conf. Ser. 4853, ed. S. L. Keil, \& S. V. Avakyan, 370
Scharmer, G. B., Narayan, G., Hillberg, T., et al. 2008, ApJ, 689, L69

Schleicher, H., Wöhl, H., \& Balthasar, H. 2003, Astron. Nachr. Suppl., 324, 114

Schlichenmaier, R., \& Collados, M. 2002, A\&A, 381, 668

Schlichenmaier, R., \& Schmidt, W. 1999, A\&A, 349, L37

Schlichenmaier, R., Bellot Rubio, L. R., \& Tritschler, A. 2004, A\&A, 415, 731

Schmidt, W., \& Schlichenmaier, R. 2000, A\&A, 364, 829

Sigwarth, M. 2001, ApJ, 563, 1031

Skumanich, A., Lites, B. W., Martínez Pillet, V., \& Seagraves, P. 1997, ApJS, 110,357

Socas-Navarro, H., Elmore, D., Pietarila, A., et al. 2006, Sol. Phys., 235, 55 Tritschler, A., Schmidt, W., Langhans, K., \& Kentischer, T. 2002, Sol. Phys., 211, 17

Tritschler, A., Schlichenmaier, R., Bellot Rubio, L. R., et al. 2004, A\&A, 415, 717

Uitenbroek, H., Miller-Ricci, E., Asensio Ramos, A., \& Trujillo Bueno, J. 2004, ApJ, 604, 960

van Noort, M., Rouppe van der Voort, L., \& Löfdahl, M. G. 2005, Sol. Phys., 228,191

von der Lühe, O., \& Kentischer, T. J. 2000, A\&AS, 146, 499

von der Lühe, O., Soltau, D., Berkefeld, T., \& Schelenz, T. 2003, in SPIE Conf. Ser. 4853, ed. S. L. Keil, \& S. V. Avakyan, 187

Wedemeyer, S., Freytag, B., Steffen, M., Ludwig, H.-G., \& Holweger, H. 2004, A\&A, 414, 1121

Wöger, F., Wedemeyer-Böhm, S., Schmidt, W., \& von der Lühe, O. 2006, A\&A, 459, L9 